\title{
ADVANCED PHOTOVOLTAIC POWER SYSTEM DEVELOPMENT AT THE U.S. AIR FORCE RESEARCH LABORATORY
}

\author{
John Merrill(1), David Wilt(1), David Chapman ${ }^{(1)}$, Geoff Bradshaw $^{(1)}$, Kyle Montgomery $^{(1)}$, Nathan Gapp ${ }^{(1)} \operatorname{and}^{(1)}$ \\ Bernie Carpenter ${ }^{(2)}$
}

\author{
(1) US Air Force Research Laboratory, 3550 Aberdeen Ave SE, KAFB, NM 87117, Email: \\ AFRL.VSSVOrgMailbox@us.af.mil \\ (2) Aerospace Corporation, Email: Bernie.F.Carpenter@aero.org
}

\begin{abstract}
Photovoltaics continue to be the primary source of electrical power for most near-Sun space missions. The desire to enhance or enable new space missions through higher efficiency, increased specific power $(\mathrm{W} / \mathrm{kg})$, increased volumetric power density $(\mathrm{W} / \mathrm{m} 3)$ and improved radiation resistance, along with decreased costs, continues to push the development of novel solar cell and array technologies. To meet present and future space power requirements, advanced multijunction solar cells and novel cell technologies are being pursued. These efforts have resulted in a continual advancement in performance, but new paradigms will be required to continue that performance trend. Similarly, new array technologies are being investigated and developed to meet the ever increasing power system performance requirements.
\end{abstract}

\section{INTRODUCTION}

Space photovoltaic (PV) development has made continual and remarkable progress, averaging roughly $0.5 \%$ per year improvement in absolute efficiency over many decades (fig. 1). Early solar cell development activities were driven solely to meet spacecraft performance needs, as PV materials costs were considered to be too high for terrestrial applications. Beginning in the early 1970's, investment in space PV development began to wane as the conventional wisdom of the day asserted that PV generation capability was limited to approximately $1 \mathrm{~kW}$ of spacecraft power and beyond that nuclear power systems would be utilized. In addition, financial forecasts indicated that photovoltaics would always be too expensive for terrestrial application. Fortunately, these apparent constraints turned out to be inaccurate, and PV development and applications have flourished for both terrestrial and spacecraft needs.

A wide variety of PV technologies have been investigated and developed in order to attain the continual efficiency improvements demonstrated, with current 1 -Sun space solar cells projected to attain $>35 \%$ efficiency in the near future. History has shown that advancements in cell efficiency, as long as the new technologies have a comparable or better environmental durability than current state of the art, are quickly adopted for widespread use in space for both commercial and government spacecraft. This speaks to the leveraging impact increases in photovoltaic system performance has on the rest of the spacecraft design.

This paper focuses on photovoltaic power system development efforts within the Space Vehicles Directorate of the Air Force Research Laboratory (AFRL) specifically focusing on advanced solar cell, blanket and array technologies. AFRL has interest and efforts in energy storage and power management technologies however this presentation will focus on the energy generation component of the power system.

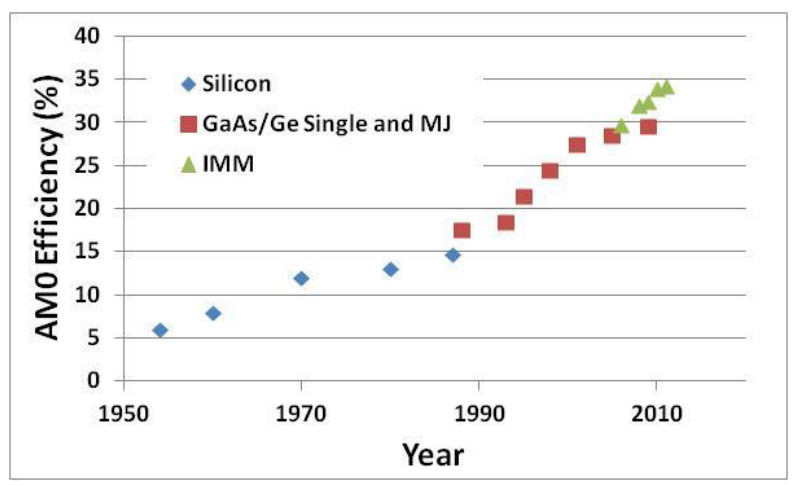

Figure 1. Historical performance trend for space solar cells.

\section{PHOTOVOLTAIC DEVICE DEVELOPMENT}

Conventional lattice matched germanium (Ge) based triple junction solar cells have largely reached their practical efficiency limit of $\sim 30 \%$. Moving to higher efficiencies has driven solar cell developers to investigate a range of alternative cell technologies, including metamorphic structures, novel materials (e.g. dilute nitride) and novel structures (e.g. quantum enhanced devices). Within the AFRL research portfolio, the emphasis in next generation cells has focused primarily on Inverted Metamorphic Multijunction (IMM) devices [1]. IMM devices consist of conventional III-V materials, but are grown inverted such that the critical high bandgap cells are produced lattice matched to the epitaxial substrate, thus preserving their high performance. The lower bandgap cells in the multjunction stack are then grown lattice 
mismatched in order to enable the optimum bandgap combinations to be achieved. This approach has resulted in air mass zero (AM0) beginning of life (BOL) efficiencies ranging from $33 \%$ to $37 \%$. AFRL/MANTECH is nearing the completion of an IMM manufacturing development program with an IMMX+ cell expected to begin AIAA S-111 qualification in the near future [2]. The IMMX+ cell offers $\sim 33 \%$ BOL efficiency and perhaps more importantly, offers a $10 \%$ performance benefit at endof-life (EOL) and at operating temperature, compared to conventional $30 \%$ class triple junction solar cells.

In addition to the performance advantage, the IMMX+ is being developed to be delivered to blanket and array manufacturers as a pre-integrated string of solar cells, known as Flex String Arrays (FSAs) (fig. 2). FSAs will be available in custom cell length strings and custom configurations and should offer significantly lower blanket and panel integration costs, compared to conventional discrete cells. A full cell repair/replacement process for the FSAs has been developed and has passed preliminary qualification confidence testing.
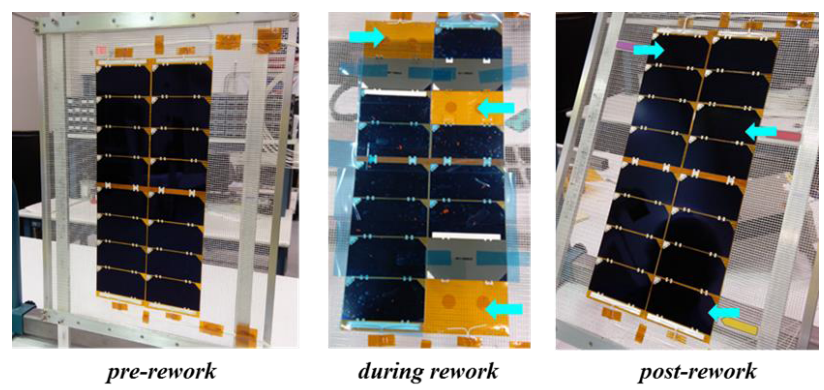

Figure 2. Four 4-cell IMM FSAs assembled into 16-cell string (l), three cells being replaced (center) and string following re-work (r) [2].

AFRL is also investigating a range of other cell technologies which offers high performance. These include dilute nitride based multijunction solar cells [3], GaAs based quantum dot photovoltaics [4], mechanically bonded multijunction solar cells [5] and solar concentrator solar cells [6]. The mechanically bonded and concentrator cells are intended for use in space solar concentrator solar arrays that will be described in the array section.

\section{CELL AND BLANKET TECHNOLOGIES}

In addition to new solar cells, AFRL is also developing ancillary technologies of benefit to a wide range of cell and array options. The first is a solar cell coverglass replacement technology known as pseudomorphic glass (PMG) [7]. PMG consists of small glass beads (e.g. fused silica) imbedded in space qualified silicone adhesive and offers a flexible, fully encapsulation alternative to conventional coverglass. PMG is currently produced in thin sheets via a doctor blade casting process, coated with a UV rejection coating (Optical Coating Solutions) and then bonded to panels once the cell strings have been integrated. This technology has been validated in space and is being used on multiple commercial LEO satellites.

With the interest of both space and terrestrial solar cell developers in thinner crystalline cells, in order to reduce mass, cost and/or increase performance, the potential for cells to fracture and lose performance may be increased. To address this potential issue, AFRL has developed an advanced solar cell metal matrix composite (MMC) metallization consisting of low-cost, low-purity, multiwall carbon nanotubes imbedded within the bulk silver metallization [8]. This technology has demonstrated the ability to electrically bridge $>40$ micron fractures in the underlying semiconductor material and was recently integrated with triple junction space solar cells. That work demonstrated that the Jsc of the cells with MMC metallization was maintained even after the cells were intentionally fractured, whereas cells with conventional metallization demonstrated $\sim 50 \%$ reduction in Jsc under similar fracturing tests.

\section{SOLAR ARRAY TECHNOLOGIES}

In contrast to the widespread adoption of new solar cell technologies, the transition of new solar array technologies has historically been very difficult. Recently however, several new array technologies have been developed which are bucking that trend and moving towards commercial acceptance.

The MOSAIC solar array, under development by Vanguard Space Technologies [9], is based on a modular (e.g. Lego-like) architecture whereby modules containing a single string of solar cells are mechanically attached to a pre-wired frame to form completed panels (fig 3). The MOSAIC frames contain internal mechanisms that enable synchronised deployment with a simple single actuator. The MOSAIC system was designed to be compatible with smaller spacecraft (e.g. ESPA class) utilizing $28 \mathrm{Vdc}$ direct energy transfer bus topology and a total power of approximately $1.5 \mathrm{~kW}$. MOSAIC arrays offer the potential for lower cost (reduced non-reoccurring engineering and custom array fabrication costs), faster development (all components are modular and can be warehoused) and the ability to test and fly new cell and panel technologies as part of the operational array. This testing feature is a new capability that is being utilized in two upcoming AFRL sponsored MOSAIC flight experiments (SurreySat and STPSAT IV). 


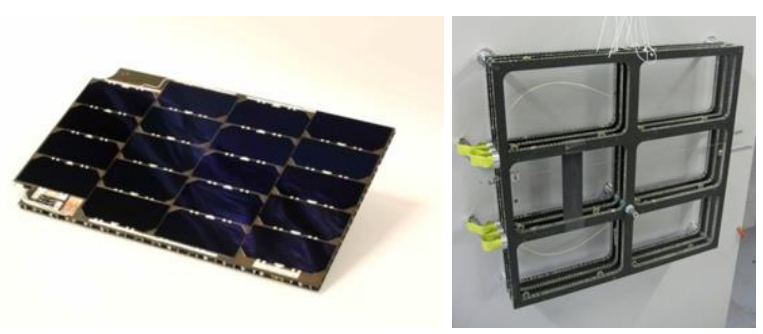

Figure 3. MOSAIC module (l) and pre-wired MOSAIC panel frame capable of holding six modules ( $r)$.

In the area of small arrays, AFRL is also working with Deployable Space Systems (DSS) on the Aladdin solar array (fig 4). This array design leverages recent advancements in strained composite technology and has the potential to offer very low cost and high reliability given the very low part count. The array consists of a strained composite structure (similar to tape measure but with a wider and flatter mid-section). In the folded configuration, cells are placed in patches separated by areas which enable the structure to fold. When the structure is flattened, it is able to be folded and stowed for launch. Once on-orbit, the array deploys using the stored strain energy in the composite structure.

The largest solar array currently being commercialized is the DSS ROSA solar array [10]. ROSA (Roll Out Solar Array) uses two strained composite slit-tube booms to deploy and tension a flexible solar array blanket. ROSA is currently under qualification development testing by Space Systems Loral (SSL) for their next generation of GEO spacecraft. In parallel with the SSL activity, AFRL is working with NASA and DSS to perform a flight demonstration of ROSA aboard the ISS (fig 5). The ISS flight experiment is primarily a structural validation of the ROSA (deployment, stiffness, etc.) although the experiment will include multiple active solar cell strings.

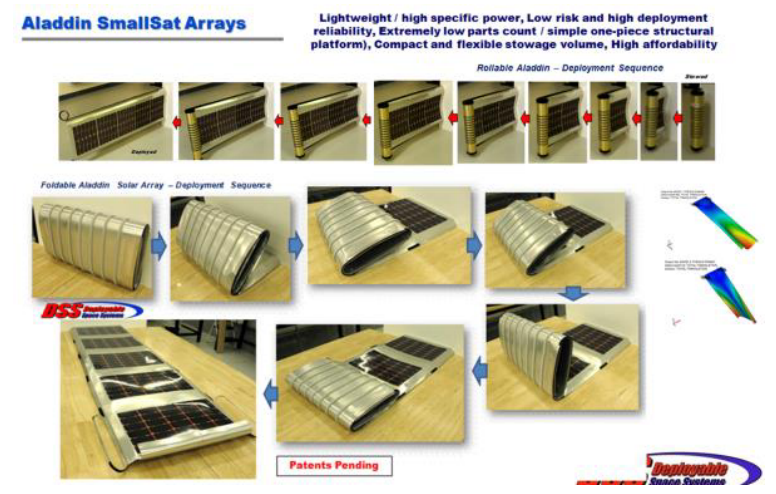

Figure 4. The DSS Aladdin solar array shown in a rolled configuration (top) and a fold-out configuration (bottom).

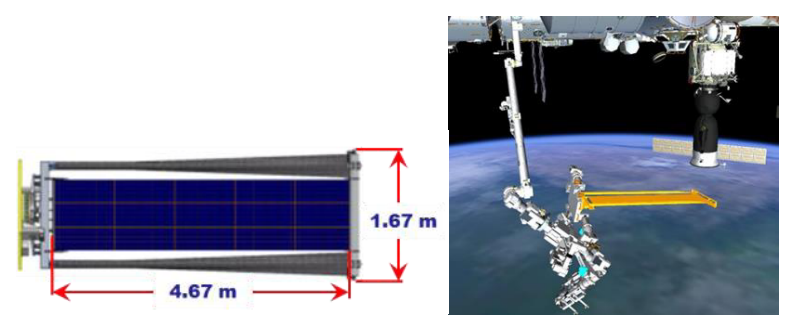

Figure 5. The DSS ROSA solar array ISS experiment (l) and shown during testing aboard the ISS at the end of the robotic arm (r).

The ROSA ISS hardware has been delivered to NASA for integration and is expected to be launched to ISS in the trunk of the DRAGON in early 2017. Once on-orbit, ROSA will be grappled by the robotic arm, deployed and characterized for approximately 7-days. The base on the ROSA experiment includes mechanical actuators to induce loads to the deployed ROSA hardware. The response of ROSA to these disturbances will be assessed using a variety of on-board sensors and photogrammetry tools. Results of the on-orbit testing will be used to validate ground models. The active solar cell strings will also be characterized via I-V testing. At the conclusion of the experiment, ROSA will be retracted and re-stowed in the DRAGON trunk which will burn up upon re-entry.

AFRL is also active in the development of solar concentrator technologies as they offer potential for cost, performance and environmental durability benefits. Current efforts are primarily considering refractive concentrator technology, given their benefits related to shape error tolerance and soiling degradation. In addition, AFRL is investigating the potential to modify high performance terrestrial concentrator technologies (Semprius) for use in space. These systems have well developed manufacturing process and utilize very small ( $\sim 600$ micron) cells enabling very thin packaging, high environmental shielding and potentially low cost. Given the past challenges of space solar concentrators and their requirements for precise pointing, these systems will have to show considerable benefit in order to transition to operational use. Fortunately there have been a number of successful uses and demonstrations of space solar concentrators, thus there is hope that this technology may be adopted in the near future.

\section{CONCLUSIONS}

Improvements in solar cell and array technology continue to transition to operational use given the tremendous benefit these technologies offer to the overall spacecraft. Rather than diminishing, the number of solar cell technologies that have the potential to provide $>30 \%$ efficiency is expanding. Given the limited size of the space marketplace, it may be challenging for all of the potential technologies to be 
commercially viable.

\section{REFERENCES}

1. Experimental results from performance improvement and radiation hardening of inverted metamorphic multi-junction solar cells, Pravin Patel; Daniel Aiken; Andreea Boca; Benjamin Cho; Daniel Chumney; Brad Clevenger; Arthur Cornfeld; Navid Fatemi; Yong Lin; James Mccarty; Fred Newman; Paul Sharps; John Spann; Mark Stan; Jeff Steinfeldt; Tansen Varghese, Photovoltaic Specialists Conference (PVSC), 2011 37th IEEE.

2. Manufacturing Improvement of Inverted Metamorphic Multijunction (IMM) Solar Cells and Flex String Array (FSA) Confidence Testing on the AFRL Mantech Program, Benjamin Cho, Daniel Derkacs , Kip Hazlett, Christopher Kerestes, Chelsea Mackos, Nathaniel Miller, Bed Pantha, Pravin Patel, Paul Sharps , Boyd Shaw, Steve Whipple, Lei Yang, Proc. $43^{\text {rd }}$ IEEE PVSC (2016)

3. Effects of in situ annealing on GaInNAs solar cells Sarah Kurtz; Richard King; Daniel Law; Aaron Ptak; John Geisz; Nasser Karam 2013 IEEE 39th Photovoltaic Specialists Conference (PVSC) Pages: 2095 - 2099

4. Intermediate band solar cell design using InAs quantum dots in AlAsSb cladding, Seth $\mathrm{M}$. Hubbard; Staffan Hellstroem; Zachary S. Bittner; Ramesh B. Laghumavarapu; Diana Huffaker, Photovoltaic Specialist Conference (PVSC), 2015 IEEE 42 ${ }^{\text {nd }}$

5. $35.8 \%$ space and $38.8 \%$ terrestrial $5 \mathrm{~J}$ direct bonded cells, P. T. Chiu; D. C Law; R. L. Woo; S. B. Singer; D. Bhusari; W. D. Hong; A. Zakaria; J. Boisvert; S. Mesropian; R. R. King; N. H. Karam, 2014 IEEE 40th Photovoltaic Specialist Conference (PVSC)

6. Six-Junction (6J) Microscale Concentrating Photovoltaics (CPV) Concepts for Space Applications, Matthew Lumb, Brent Fisher, Ken Schmieder, Matthew Meitl, Proc. $43^{\text {rd }}$ IEEE PVSC (2016)

7. Novel flexible solar cell coverglass for space photovoltaic devices, David Wilt; Neil Snyder; Phillip Jenkins; Andy Gray, 2013 IEEE 39th Photovoltaic Specialists Conference (PVSC)

8. Silver-Carbon-Nanotube Metal Matrix Composites for Metal Contacts on Space
Photovoltaic Cells, Omar K. Abudayyeh; Nathan D. Gapp; Cayla Nelson; David M. Wilt; Sang M. Han, IEEE Journal of Photovoltaics, Year: 2016, Volume: 6, Issue: 1, Pages: 337 - 342

9. Increasing the TRL level of new PV technologies using modular solar panels, N. Walmsley; T. Stern; D. Wilt, Photovoltaic Specialists Conference (PVSC), 2012 38th IEEE

10. Commercialization of Deployable Space Systems' roll-out solar array (ROSA) technology for Space Systems Loral (SSL) solar arrays, Bao Hoang; Steve White; Brian Spence; Steven Kiefer, 2016 IEEE Aerospace Conference 